\title{
Influence of Long-Term Sensitization on Long-Term Habituation of the Acoustic Startle Response in Rats: Central Gray Lesions, Preexposure, and Extinction
}

\author{
George S. Borszcz, Jacquelyn Cranney, and Robert N. Leaton \\ Dartmouth College
}

\begin{abstract}
The relation between long-term decrements of the acoustic startle response in rats and the development of freezing behavior during habituation training was examined. Freezing behavior developed over the initial trials of habituation training, and the rate of long-term response decrements was found to be inversely related to the development of freezing. Manipulations (neurological or behavioral) that either reduced the level of freezing or retarded its development promoted startle response decrements. In Experiment 1, rats receiving electrolytic lesions of the ventrolateral periaqueductal gray demonstrated both accelerated long-term startle response decrements and retarded development of freezing behavior. In Experiment 2, preexposure to the startle apparatus (i.e., latent inhibition) accelerated long-term startle decrements and inhibited development of freezing. In Experiment 3, exposure to the startle apparatus following initial habituation training (i.e., extinction) reduced both freezing behavior and startle response amplitudes. The results are discussed in terms of the influence of Pavlovian fear conditioning on longterm habituation of the acoustic startle response.
\end{abstract}

Pavlovian fear conditioning has long been known to influence the amplitude of the acoustic startle response in rats. Using the potentiated startle paradigm, a number of investigators have shown that startle amplitudes are augmented when the startle stimulus is presented in conjunction with a conditional stimulus previously paired with footshock (Brown, Kalish, \& Farber, 1951; Davis \& Astrachan, 1978; Kurtz \& Siegel, 1966). This potentiation of acoustic startle is believed to reflect the general response-energizing effects associated with the conditioning of a central fear state (Brown, 1939; Brown et al., 1951). Consistent with these findings, the degree to which startle amplitudes are potentiated has been shown to be directly related to the proportion of time spent freezing by animals prior to presentation of the startle stimulus (Leaton \& Borszcz, 1985). The freezing response, characterized by crouching and immobility, represents a conditional reaction of rats to conditional stimuli associated with aversive events (Blanchard \& Blanchard, 1969; Bolles \& Collier, 1976; Fanselow, 1980).

We have noted, as have others (e.g., Korn \& Moyer, 1966; Marlin \& Miller, 1981), that during the early course of habituation training to an acoustic startle stimulus, rats exhibit freezing behavior. The influence of freezing on the rate of acoustic startle decrements has not, however, been systematically examined. Given the direct relation between acoustic startle response amplitudes and freezing behavior, startle decrements might be influenced by the freezing exhibited by

This article is based on a doctoral dissertation prepared by George S. Borszcz in the Department of Psychology, Dartmouth College.

Jacquelyn Cranney is currently at the School of Psychology, The University of New South Wales, Kensington, New South Wales, Australia.

Correspondence concerning this article should be addressed to George S. Borszcz, Department of Psychology, Dartmouth College, Hanover, New Hampshire 03755. animals during habituation training. Furthermore, assuming that freezing during habituation training reflects fear conditioning, manipulations that interfere with or reduce such conditioning also would be expected to affect startle decrements. In the present study we outline the development of freezing behavior during long-term habituation training and examine the relation between long-term startle decrements and the freezing response.

\section{Experiment 1}

If during habituation training, acoustic startle response amplitudes are related to the freezing behavior that rats exhibit, then brain areas that are known to affect freezing and/ or fear conditioning should influence response decrements. Specifically, brain lesions that have been shown to reduce freezing and fear conditioning would be expected to facilitate long-term startle response decrements by reducing the response-amplifying effects associated with conditioned fear. One such brain area is the ventrolateral aspect of the midbrain periaqueductal gray (vPAG), which has been shown to mediate a number of aversively motivated tasks (Halpern, 1968; Liebman, Mayer, \& Liebeskind, 1970; Lyon, 1964). Of particular interest is the observation that lesions restricted to the vPAG inhibit freezing to contextual cues previously paired with footshock (Liebman et al., 1970). In Experiment 1 we examined the influence of vPAG lesions (unilateral and bilateral) on long-term acoustic startle response decrements and on the development of freezing behavior during habituation training.

\section{Method}

Subjects. Thirty-six male Sprague-Dawley-derived albino rats, approximately 110 days old and weighing between $380 \mathrm{~g}$ and $430 \mathrm{~g}$, were used. They were individually housed, maintained on a 14-hr:10- 
$\mathrm{hr}$ light/dark cycle, and allowed free access to food and water in their home cages throughout the experiment. Prior to surgery, animals were assigned equally to three groups matched on the basis of their body weights: sham, unilateral, and bilateral.

Surgery. Surgery was performed under sodium pentobarbital anesthesia $(50 \mathrm{mg} / \mathrm{kg})$ under clean but not aseptic conditions. Lesions of the vPAG were made electrolytically with an anodal current of 1 $\mathrm{mA}$ for a duration of $5 \mathrm{~s}$. Electrodes were stereotaxically oriented according to the atlas of Pellegrino and Cushman (1967); A/P = -5.4 , Lat. $= \pm 0.5, \mathrm{D} / \mathrm{V}=6.0$. Sham lesions consisted of lowering electrodes to these coordinates without passing current. A headholder similar to that described by Frommer (1971) was used to avoid damage to the rats' tympanic membranes. Electrodes were constructed of 27 -ga stainless steel hypodermic tubing insulated except for $0.5 \mathrm{~mm}$ at the tip.

Anatomical procedures. After the experiments, animals were intracardially perfused with normal saline followed by $10 \%$ formalin. Frozen sections were cut at $40 \mu \mathrm{m}$, and every third section was saved and stained by a modified Klüver-Barrera method. For evaluating the extent of brain damage, the stained sections were projected onto brain diagrams taken from the atlas of Pellegrino and Cushman (1967). Damage was traced and reconstructed on diagrams separated by $0.2 \mathrm{~mm}$ throughout the full extent of the lesion. The vPAG was defined as the portion of the periaqueductal gray (PAG) lying below the level of the cerebral aqueduct, an area corresponding to the ventral cellular subdivision of the PAG (Beitz, 1982).

Apparatus. Similar startle apparatus has been described previously (Leaton, 1976). Animals were tested in one of two identical 20$\mathrm{cm} \times 12-\mathrm{cm} \times 14-\mathrm{cm}$ startle chambers with floors, walls, and ceiling constructed of $2.5-\mathrm{mm}$ steel rods mounted within a Plexiglas frame. This chamber was sandwiched between compression springs that were attached to a rigid superstructure. Vertical displacement of the chamber moved an attached magnet within a fixed coil, inducing a voltage that was digitized, rectified, and integrated by a microcomputer system. Startle amplitude was taken as the integrated voltage for the 200 -ms epoch beginning at onset of the startle stimulus. In addition to the stimulus-provoked startle response, the movement of the startle chamber was monitored in 1-s intervals for the $10-\mathrm{s}$ period prior to each startle stimulus. To detect very slight movements of the startle chamber, the microcomputer increased the sensitivity of the measure during this 10-s sampling period. A criterion response of 0 or 1 in the integrated voltage output for a 1-s interval was taken as an index of freezing behavior, and percentage of freezing was calculated as the proportion of 0 and 1 responses in the 10 one-s intervals prior to onset of the startle stimulus. In preliminary experiments, this index of freezing correlated highly $(r=.92)$ with a time-sampling measure of freezing (Fanselow, 1981) in which the rats' behavior was judged as either freezing or active every $2 \mathrm{~s}$.

Each startle chamber was enclosed within a separate sound-attenuating box. The startle stimulus, a 95-dB (SPL), 100-ms white noise burst, was delivered through a 9-cm piezoelectric tweeter centered 12 $\mathrm{cm}$ from the long wall of the startle chamber. Startle stimuli were superimposed upon a continuous white noise background (70 $\mathrm{dB})$ provided by a small second speaker. Auditory intensities were measured with a General Radio sound-level meter (Type 1551-C, 20-kHz setting) with the microphone centered inside the startle chamber.

Procedure. A minimum of 10 days following surgery, animals began habituation training every other day for a total of 4 days. The first day of training was staggered so that half of the animals in each group were tested each day. On each training day there were three sessions, with an intersession interval of $2 \mathrm{hr}$ and $30 \mathrm{~min}$. Animals were returned to their home cages for the duration of the intersession interval. Each session consisted of 10 presentations of the startle stimulus on a $60-\mathrm{s}$ interstimulus interval. The first stimulus presentation of each session occurred $2 \mathrm{~min}$ and $30 \mathrm{~s}$ after the animals were placed in the startle chamber.

\section{Results}

Anatomy. Diagrammatic cross-sections of the lesion of a representative animal from the bilateral group are shown in Figure 1. Unilateral lesions occupied the same rostrocaudal extent of the VPAG, with the majority of damage restricted to one side of the midline. The rostrocaudal extent of the lesions occupied 0.6-1.2 mm (mean $0.82 \mathrm{~mm}$ ) of the PAG, with lesions extending rostrally as far as the caudal limit of the nucleus ruber and as far caudally as the caudal extent of the stratum album mediale of the superior colliculus. Damage was restricted to the ventral and lateral subdivisions of the PAG, with more than $50 \%$ of the damage occurring below the level of the cerebral aqueduct. Typically included in these lesions were portions of the dorsal raphe nucleus, trigeminal nucleus, occulomotor nucleus, medial longitudinal fasciculus, and reticular formation surrounding the vPAG. Except for electrode tracts, damage was not observed in either the dorsal subdivision of the PAG or the overlying tectum.

General behavior. Recovery from surgery was uneventful. None of the animals exhibited either the chronic weight loss or disruption of grooming that had been reported to accompany large lesions of the PAG (Liebman et al., 1970).

Startle response. Figure 2A shows the mean startle amplitudes on the first trial of each session for all three groups. (In this and all subsequent experiments, session means showed the same overall pattern and statistical outcomes but obscured the possible influence of experimental treatments on the initial responsiveness of animals to the startle stimulus.) Repeatedmeasures analysis of variance of first-trial startle amplitudes of the three groups over the 12 sessions of habituation training revealed significant main effects of group, $F(2,33)=4.01, p$ $<.05$, and session, $F(11,363)=30.24, p<.01$, as well as a significant Group $\times$ Session interaction, $F(22,363)=2.15, p$ $<.01$. Comparisons of either lesioned group with the sham group revealed significant group differences (all $p s<.05$ ) and significant Group $\times$ Session interactions [sham vs. unilateral, $F(11,242)=1.88, p<.05$; sham vs. bilateral, $F(11,242)=$ $3.95, p<.01$ ], indicating facilitated startle decrements in lesioned groups relative to sham operated controls. The two lesioned groups did not differ significantly on any measure.

The nature of the differences in responsiveness between the sham and lesioned groups is most evident on Day 1 of habituation training. Overall analysis of first-trial startle amplitudes over the three sessions of Day 1 yielded significant main effects of group, $F(2,33)=3.72, p<.05$, and session, $F(2,66)=15.11, p<.01$, and a Group $\times$ Session interaction, $F(4,66)=3.69, p<.01$. This interaction reflects the finding that both lesioned groups showed a significant decrement in first-trial startle amplitudes across sessions on Day 1 [unilateral $F(2,22)=8.63, p<.01$; bilateral, $F(2,22)=17.75, p<$ $.01]$, whereas the sham group did not change significantly across these trials, $F(2,22)<1.0$. Groups did not differ in responsiveness to the initial startle stimulus (Trial 1, Session 1), $F(2,33)<1.0$.

The differences in first-trial startle amplitudes that developed between lesioned and nonlesioned groups on Day 1 decreased over the last 3 days of habituation training. By Day 4 , groups neither differed from one another, $F(2,33)<1.0$, nor did any group show a decrement in responding over sessions (all ps > .30). 


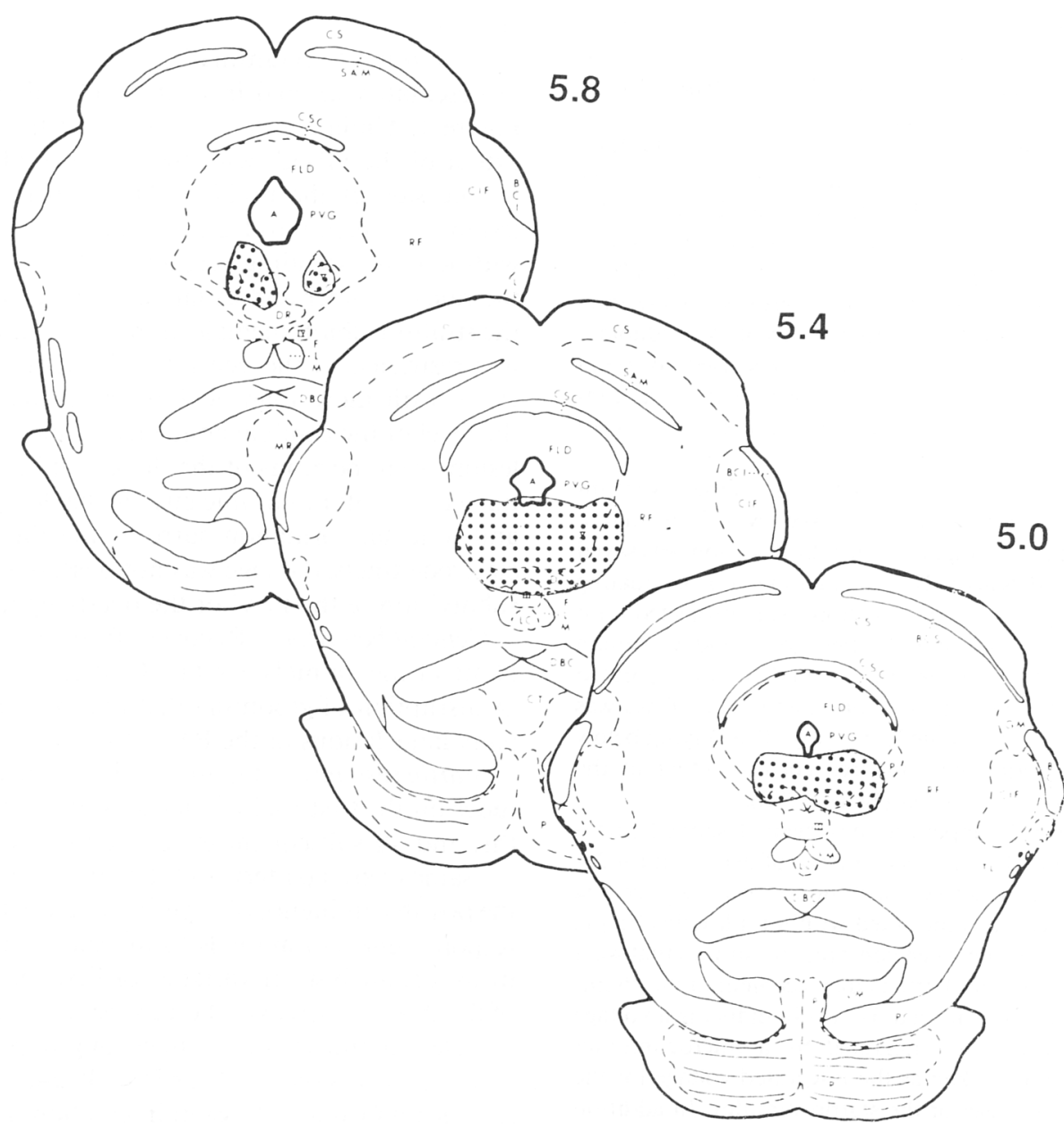

Figure 1. Diagrammatic cross-sections of a ventrolateral periaqueductal gray (vPAG) lesion from a representative animal of the bilateral lesioned group. (Unilateral lesions occupied the same rostrocaudal extent of the vPAG, with the majority of damage restricted to one side of the midline. Coordinates are from the atlas of Pellegrino and Cushman, 1967, and represent distance [in mm] from bregma.)

Analysis of the 10 startle amplitudes within each session revealed that no significant reductions in startle response amplitudes occurred within sessions. Apparently the relatively long interstimulus interval $(60 \mathrm{~s})$ used in the present study could not produce within-session startle response decrements. Alternatively, the limited number of trials within each session were insufficient to permit observation of within-session decrements that develop with the startle stimulus parameters employed.

Freezing. Figure 2B shows mean percentage of freezing prior to the first trial of each session for each group. (In this and all subsequent experiments, session means showed the same overall pattern and statistical outcomes but obscured the possible influence of experimental treatments on percentage of freezing before presentation of the initial startle stimulus.) Overall analysis of first-trial freezing scores of the three groups over the 12 sessions of habituation training revealed a significant main effect of session, $F(11,363)=11.02, p<.01$, and a significant Group $\times$ Session interaction, $F(22,363)=$ $1.7, p<.05$. Comparison of either lesioned group with the sham group also resulted in significant Group $\times$ Session interactions [sham vs. unilateral, $F(11,242)=1.86, p<.05$; sham vs. bilateral, $F(11,242)=2.83, p<.01]$. The two lesioned groups did not differ significantly on any measure.

The nature of the differences between groups is again most evident on Day 1. Overall analysis of first-trial percentage of freezing scores over the three sessions on Day 1 revealed significant main effects of group, $F(2,33)=4.41, p<.05$, and session, $F(2,66)=47.86, p<.01$, and a significant Group $\times$ Session interaction, $F(4,66)=5.26, p<.01$. This interaction reflects the finding that although all groups showed a significant increase in first-trial freezing over sessions on Day 1 (all $p s<.05$ ), the increase exhibited by the sham group was greater than that shown by either lesioned group [Group $X$ Session interaction: sham vs. unilateral, $F(2,44)=6.47, p<$ .01 ; sham vs. bilateral, $F(2,44)=10.99, p<.01$ ]. Groups did not, however, differ significantly from one another in percentage of freezing before presentation of the initial startle stimulus, $F(2,33)<1.0$.

The differences in percentage of freezing that developed between lesioned and sham groups on Day 1 decreased over the last 3 days of habituation training. By Day 4, groups 

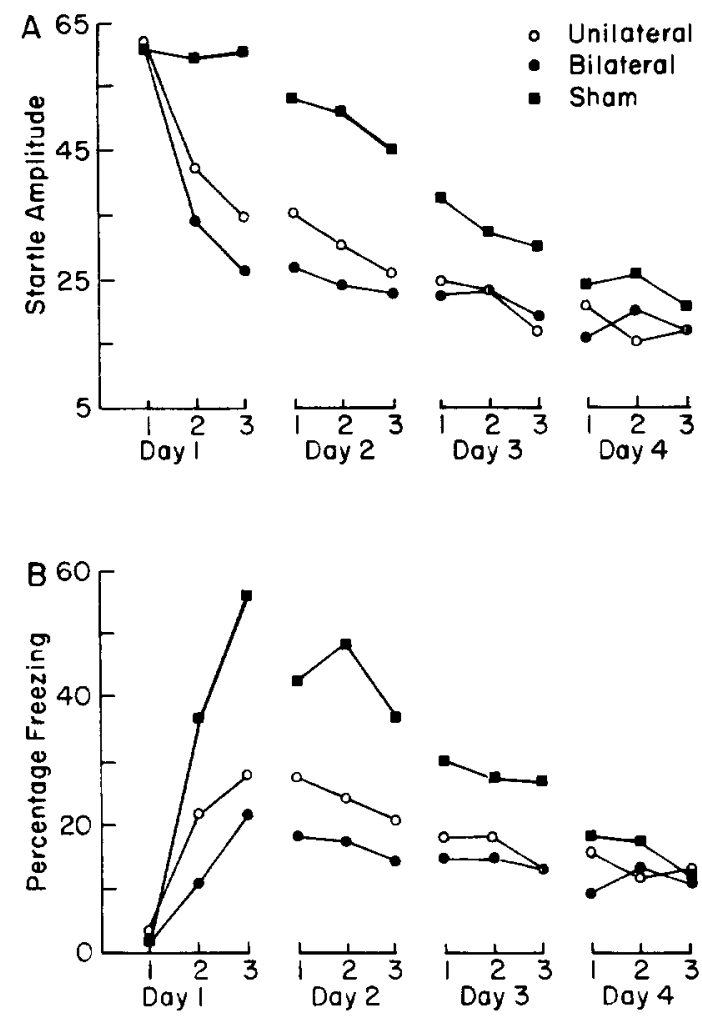

Figure 2. Comparison from Experiment 1 of startle amplitudes with percentage of freezing that preceded each startle stimulus. Panel A: Mean first-trial startle amplitudes for the sham, unilateral, and bilateral lesioned groups on the 12 sessions of habituation training. Panel B: Mean first-trial percentage of freezing for the sham, unilateral, and bilateral lesioned groups on the 12 sessions of habituation training.

neither differed from one another, $F(2,33)<1.0$, nor did any group show a change in percentage of freezing over sessions (all $p \mathrm{~s}>.50$ ).

Changes in percentage of freezing observed on first-trial scores were reflected by changes in freezing behavior that occurred within sessions. Increases and decreases in percentage of freezing that were observed from one first-trial point to the next could be predicted by within-sessions changes in percentage of freezing. For example, the increase in percentage of freezing observed on first-trial scores across the three sessions on Day 1 also was evident within sessions. Repeatedmeasures analysis of variance of the 10 freezing scores of each session on Day 1 revealed that all groups showed an orderly increase in freezing within Session 1 (all $p s<.01$ ). Unilateral and sham groups continued to show a significant increase in freezing within Session $2(p s<.05)$.

\section{Discussion}

The results of Experiment 1 demonstrated that long-term acoustic startle response decrements are inversely related to the development of freezing behavior during habituation training. Relative to sham-operated controls, animals with vPAG lesions exhibited an accelerated rate of long-term acoustic startle decrements that was accompanied by retarded development of freezing. These group differences in startle responding and percentage of freezing were not evident on the initial trial of habituation training but developed across sessions on Day 1. This relation between percentage of freezing and startle amplitude was evident not only between groups but also within groups. Correlations of mean first-trial percentage of freezing across the first three sessions of Day 1 with the rate of startle habituation (defined as the exponent derived from fitting data to the negative exponential equation, $\log y$ $=\log \mathrm{K}+\mathrm{A} \log \mathrm{N}$, by a least squares analysis; see Leaton, 1976) over those same trials proved significant: sham $r(10)=$ .93 ; unilateral $r(10)=.80$; bilateral $r(10)=.91$; and overall $r(34)=.79$. Group differences on both measures also were evident on the first trial on Day 2 of habituation training. The development and maintenance of these group differences over extended intersession intervals (i.e., $2.5 \mathrm{hr}$ between sessions; $40.5 \mathrm{hr}$ between days) indicate a long-term relation between percentage of freezing and startle amplitudes. As habituation training continued, group differences on both measures waned to the extent that by the end of training groups no longer differed on either measure.

The relation between percentage of freezing and startle amplitude observed during habituation training is similar to that previously reported to occur in the potentiated startle paradigm (Leaton \& Borszcz, 1985). The degree to which prior fear conditioning enhanced acoustic startle amplitudes was found to be directly related to the proportion of time spent freezing by animals before presentation of the startle stimulus. To the extent that the development of freezing during habituation training also reflects fear conditioning, the inverse relation between acoustic startle response decrements and percentage of freezing may reflect fear-induced enhancement of startle responding. That is, freezing may be indicative of a response-energizing process associated with habituation training that masks long-term startle response decrements. Lesions of brain areas, such as the vPAG, that inhibit fear conditioning may facilitate startle decrements by limiting the response-energizing effects that accompany the fear state.

Although lesioned groups did not differ significantly on any measure, there was a tendency for the bilateral group to freeze less and habituate more quickly than the unilateral group. This trend may either be indicative of an effect of laterality within a midline structure such as the PAG or an effect simply related to the extent of damage to the vPAG. It has been reported that the degree to which freezing behavior is disrupted following lesions of the PAG is proportional to the amount of damage to the vPAG (Liebman et al., 1970).

\section{Experiment 2}

The finding in Experiment 1 of an orderly development of freezing during the early course of habituation training and the maintenance of freezing over long intersession intervals suggests that freezing may reflect Pavlovian fear conditioning to the contextual cues (conditional stimuli, CS) of the startle apparatus. The unconditional stimulus (UCS) supporting fear conditioning is not known; confinement to the stabilimeters, placement in and removal from the stabilimeters, and the startle stimulus itself may all be aversive to the animal. 
However, the observations that freezing in Experiment 1 did not develop until after presentation of the initial startle stimulus and that freezing showed an orderly increase within sessions on Day 1 suggests that the startle stimulus may serve as the principal UCS.

If freezing during habituation training reflects Pavlovian fear conditioning to contextual cues of the startle apparatus, then behavioral manipulations that retard this conditioning should reduce development of the freezing response. By retarding fear conditioning, the startle response amplifying effects that accompany habituation training would presumably be attenuated and, as with VPAG lesions in Experiment 1, long-term startle decrements would be accelerated. One behavioral manipulation that reliably retards conditioning is latent inhibition (Lubow \& Moore, 1959; Lubow, Rifkin, \& Alek, 1976), which involves nonreinforced exposure to the CS prior to its pairing with the UCS. In a habituation training paradigm, latent inhibition would consist of preexposure to the startle apparatus (i.e., CS) prior to the presentation of the startle stimulus (i.e., UCS). In Experiment 2 we examined the influence of preexposing animals to the startle apparatus on the development of freezing and on the rate of acoustic startle decrements.

Animals in Experiment 1 had not been preexposed or handled prior to habituation training. The usual procedure in this laboratory that generates rapid long-term startle decrements (e.g., Leaton, 1976) in control groups is to both preexpose and handle animals. Because handling covaries with preexposure in the usual paradigm and could lead to changes in the development of freezing, a nonpreexposed-handled group was included in this experiment. In order to determine whether the simple exposure of animals to the startle apparatus unconditionally elicits freezing, freezing was monitored during preexposure to the startle apparatus.

\section{Method}

Subjects. Twenty-four male Sprague-Dawley-derived albino rats, approximately 150 days old and weighing between $420 \mathrm{~g}$ and $480 \mathrm{~g}$, were used. They were individually housed and maintained in a manner similar to animals in Experiment 1. Prior to experimentation, animals were assigned equally to three groups matched on the basis of their body weights: NIV (naive), HNL (handled), and PRE (preexposed).

Apparatus and stimuli. The startle apparatus and startle-eliciting stimuli ( $95 \mathrm{~dB}, 100-\mathrm{ms}$ white noise) were identical to that used in the previous study.

Procedure. Prior to habituation training, the three groups of animals were treated differently. On 5 consecutive days, both the HNL and PRE groups were handled twice daily for $30 \mathrm{~s}$ each. On the next 4 consecutive days, the PRE animals were preexposed to the startle apparatus for $5 \mathrm{~min}$ each day, and their freezing behavior was monitored each minute for $10 \mathrm{~s}$ beginning $50 \mathrm{~s}$ after placement into the startle chamber. During these $\mathbf{4}$ days the HNL group was handled once a day for $30 \mathrm{~s}$. The NIV group remained in their home cages throughout this period and were neither handled nor preexposed to the startle apparatus. After the different pretreatments, all animals were given habituation training every other day for a total of 4 days. Habituation training was identical to that described in Experiment 1.

\section{Results}

Startle response. Figure $3 \mathrm{~A}$ shows the mean acoustic startle response amplitudes on the first trial of each session for all three groups. Because of equipment failure, data of 2 NIV animals were dropped from the data analysis. The NIV and HNL groups did not differ significantly on any analysis and were combined into a nonpreexposed group (NON-PRE) for comparisons with the PRE group. Overall analysis of first trial startle amplitudes of the two groups over the 12 sessions of habituation training revealed a significant main effect of session, $F(1,20)=7.08, p<.01$, and a significant Group $\times$ Session interaction, $F(11,220)=2.51, p<.01$. These results reflect the increased rate of startle response decrement in the PRE group relative to the NON-PRE group.

Development of differences among groups in startle responding are most clearly revealed on Day 1 of habituation training. Analysis of first-trial startle amplitudes of the NONPRE and PRE groups over the three sessions of Day 1 yielded a significant Group $\times$ Session interaction, $F(2,40)=9.91, p$ $<.01$. This interaction reflects the finding that only the PRE group showed a significant decrement in startle amplitudes across sessions on Day 1 [PRE, $F(2,14)=13.29, p<.01$; NON-
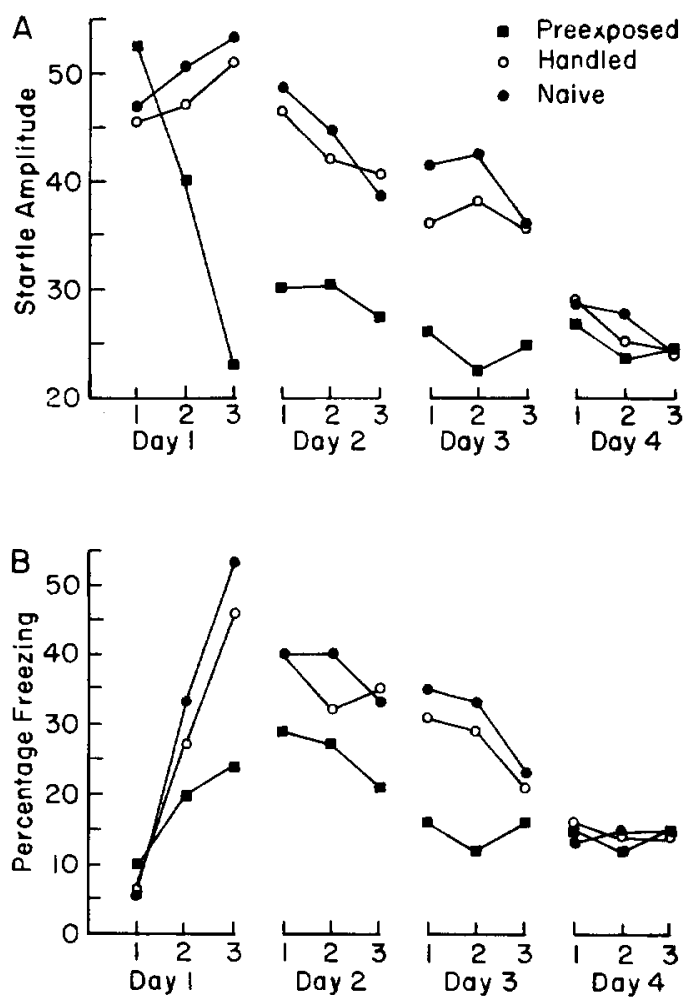

Figure 3. Comparison from Experiment 2 of startle amplitudes with percentage of freezing that preceded each startle stimulus. Panel A: Mean first-trial startle amplitudes for the preexposed, handled, and naive groups of the 12 sessions on habituation training. Panel B: Mean first-trial percentage of freezing for the preexposed, handled, and naive groups on the 12 sessions of habituation training. 
PRE, $F(2,26)<1.0$ ]. Groups did not differ in their responsiveness to the initial startle stimulus, $F(1,20)<1.0$.

The differences in startle amplitudes that developed between PRE and NON-PRE groups on Day 1 decreased over the last 3 days of habituation training. By Day 4, groups neither differed from one another, $F(1,20)<1.0$, nor did either group show a decrement in responding over sessions (all $p \mathrm{~s}>$ .25).

As in Experiment 1, analysis of the 10 startle amplitudes within each session revealed that no significant reductions in startle response amplitudes occurred within sessions.

Freezing. Figure 3B shows mean percentage of freezing prior to the first trial of each session for each group. The NIV and HNL groups did not differ significantly on any analysis and are combined into a NON-PRE group for comparisons with the PRE group. Overall analysis of first-trial freezing scores on the 12 sessions of habituation training revealed a significant main effect for session, $F(11,220)=11.26, p<.01$, and a significant Group $\times$ Session interaction, $F(11,220)=2.04, p$ $<.05$. This interaction reflects the greater development of freezing behavior in the NON-PRE group relative to the PRE group.

Again the development of differences among groups is most evident on Day 1. Analysis of first-trial percentage of freezing scores of the NON-PRE and PRE groups on Day 1 revealed a significant main effect for session, $F(2,40)=31.80, p<.01$, and a significant Group $\times$ Session interaction, $F(2,40)=$ $6.28, p<.01$. This interaction reflects the finding that atthough the NON-PRE and PRE groups showed a significant increase in freezing over sessions on Day 1 (all $p s<.05$ ), the increase exhibited by the NON-PRE group was greater than that shown by the PRE group. Groups did not differ from one another in percentage of freezing prior to the initial startle stimulus on Day $1, F(1,20)=1.98, p>.05$.

The differences that developed between PRE and NON-PRE groups in freezing on Day 1 decreased over the last 3 days of habituation training. By Day 4 groups neither differed from one another, $F(1,20)<1.0$, nor did either group show a change in percentage of freezing over sessions $(p s>.85)$.

Percentage of freezing exhibited by both groups prior to presentation of the initial startle stimulus on Day 1 did not differ from that exhibited by the PRE group during preexposure (data not shown). Over the course of preexposure to the startle apparatus, the PRE group showed a mean of $<5 \%$ of freezing during any one mock-trial sampling period and showed no tendency to change freezing behavior within or between preexposure sessions.

As in Experiment 1, changes in percentage of freezing observed on first-trial scores could be predicted by withinsession changes in percentage of freezing. For example, the increase in percentage of freezing observed on first-trial scores across the three sessions on Day 1 also was evident within sessions. Repeated-measures analysis of variance of the 10 freezing scores of each session on Day 1 revealed that PRE and NON-PRE groups showed an orderly increase in freezing within Session $1(p s<.05)$. The NON-PRE group continued to show a marginally significant increase in percentage of freezing across Session $2(p<.057)$.

\section{Discussion}

The results of Experiment 2 are similar to those of Experiment 1 , except for the substitution of animals with vPAG lesions for animals preexposed to the startle apparatus in the present study. Although there were no differences between preexposed and nonpreexposed groups in responsiveness to the initial startle stimulus, the preexposed group showed enhanced long-term startle decrements over the 12 sessions of habituation training relative to the nonpreexposed group. The rate of startle decrement also was found to be inversely related to the development of freezing during long-term habituation training. The preexposed group demonstrated an accelerated rate of startle decrement, but it also exhibited retarded development of the freezing response. As in Experiment 1 , mean first-trial percentage of freezing on Day 1 was highly correlated with the rate of startle decrement, overall $r(22)=.84$.

The results of Experiment 2 are consistent with the hypothesis that development of freezing during long-term habituation training reflects Pavlovian fear conditioning and that such conditioning retards startle decrements by inflating startle amplitudes. As demonstrated by both Experiments 1 and 2, manipulations designed to retard fear conditioning during habituation training were found to reduce freezing and facilitate startle decrements. Preexposure to the startle apparatus is believed to retard fear conditioning through the latent inhibition of apparatus cues that serve as the conditional stimulus for fear conditioning during habituation training.

\section{Experiment 3}

Another test of the hypothesis that fear conditioning during habituation training opposes startle decrements by inflating startle amplitudes would be to examine the effects of extinguishing fear on startle responding. If the differences in startle decrements between preexposed and nonpreexposed groups that developed by the end of Day 1 in Experiment 2 were the result of the differential fear conditioning, then extinguishing fear in the nonpreexposed group should eliminate or reduce group differences in startle amplitudes. In Experiment 3 we tested this hypothesis by giving preexposed and nonpreexposed groups extinction training after Day 1 of habituation training. Extinction sessions involved exposing animals to the startle apparatus (CS) without presenting the startle stimulus (UCS). If our analysis is correct, these sessions should extinguish Pavlovian fear conditioning to the contextual cues of the startle apparatus, reduce freezing, and reduce startle amplitudes.

\section{Method}

Subjects. Forty male albino Spague-Dawley-derived rats, approximately 150 days old and weighing between $430 \mathrm{~g}$ and $510 \mathrm{~g}$, were housed in a similar manner to subjects in Experiments 1 and 2. Animals were assigned equally to two groups matched on the basis of body weights: NON-PRE (nonpreexposed) and PRE (preexposed).

Apparatus and stimuli. The apparatus was identical to that used in Experiment 2, with the addition of a second "preexposure" context. 
This second context consisted of well-lit yellow plastic cylindrical trash bins $38 \mathrm{~cm}$ high and $34 \mathrm{~cm}$ in diameter, with white lattice lids. As in the startle apparatus, continuous white noise $(70 \mathrm{~dB})$ masked extraneous auditory stimuli. Because preexposed animals in Experiment 2 showed asymptotic startle responding by the last trial of Day 1 of habituation training, the intensity of the startle stimulus was increased to $105 \mathrm{~dB}$ to avoid possible floor effects in startle amplitude.

Procedure. Prior to habituation training, PRE and NON-PRE groups were treated in a manner similar to Experiment 2. On 5 consecutive days, both the PRE and NON-PRE groups were handled twice a day for $30 \mathrm{~s}$ each. During the next 4 consecutive days, the PRE animals were placed in the startle apparatus for 5 min each day, and freezing was monitored as in Experiment 2. The exception with Experiment 2 was that the NON-PRE animals were placed in novel plastic bins on the days PRE animals were preexposed to the startle apparatus. Following pretraining, habituation training was initiated on alternate days as described in Experiment 2. On the basis of their mean startle amplitudes (first-trial and session means) on Day 1 of habituation training, the PRE and NON-PRE groups were each divided into two matched groups of 10 animals each: PE (preexposed-extinguished), PN (preexposed-nonextinguished), NE (nonpreexposed-extinguished), and NN (nonpreexposed-nonextinguished). Following Day 1 of habituation training, animals were exposed to one of two different contexts (extinction day). The PE and NE groups were given three sessions of startle apparatus exposure. Session length ( $12 \mathrm{~min} 30 \mathrm{~s}$ ) and intersession intervals were equivalent to that of habituation training, but no startle stimulus was presented. Freezing behavior was monitored on the same schedule as that of Day 1 of habituation training. The PN and NN groups were placed in the plastic bins on the same session schedule; freezing was not monitored in these animals at this time.

On alternate days beginning after the Extinction Day, all animals were again given habituation training for 2 days on the same schedule as Day 1. Freezing was monitored for all animals during habituation training.

\section{Results}

Startle response. Figure 4A shows the mean startle amplitudes on the first trial of each session and on the last trial of Day 1 for all four groups. Analysis of first-trial startle amplitudes of the combined PRE and combined NON-PRE groups on Day 1 replicated Experiment 2. There was a significant Group $\times$ Session interaction, $F(2,76)=3.76, p<.05$, reflecting the finding that only the PRE group exhibited a significant decrement in startle responding across sessions on Day 1 [PRE, $F(2$, $38)=4.80, p<.05$; NON-PRE, $F(2,38)<1.0]$. The PRE and NON-PRE groups did not differ in responding to the initial startle stimulus presented on Day $1, F(1,38)<1.0$. However, by the last trial on Day 1, PRE and NON-PRE groups did differ significantly in startle amplitude, $F(1,38)=6.72, p<.05$.

The most sensitive index of the impact of the extinction manipulation on startle responding was the change in amplitude from the last trial of Day 1 to the first trial after extinction training (Trail 1, Day 3). This comparison is shown in Figure 4A. Overall analysis of these data points yielded a significant Group $\times$ Trial interaction, $F(3,36)=3.08, p<.05$. Analysis of this interaction revealed that whereas the NE groups decreased responding between the last trial of Day 1 and the first trial of Day $3, t(9)=5.84, p<.01$, none of the other groups showed a significant change in startle amplitudes (all ps $>$.20).
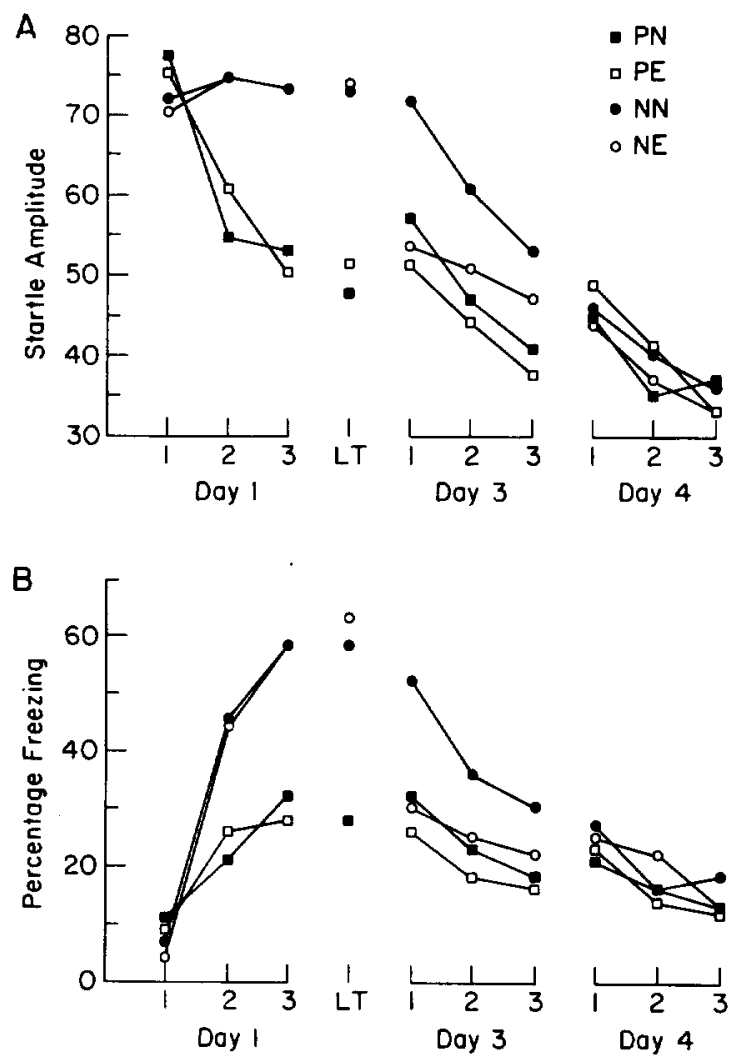

Figure 4. Comparison from Experiment 3 of startle amplitudes with percentage of freezing that preceded each startle stimulus during habituation training. (Extinction training occurred on Day 2 [data shown in Figure 5].) Panel A: Mean startle amplitudes on the first trial of each session and on the last trial of Day 1 of habituation training for the preexposed-nonextinguished (PN), preexposed-extinguished (PE), nonpreexposed-nonextinguished (NN), and nonpreexposed-extinguished (NE) groups. Panel B: Mean percentage of freezing on the first trial of each session and on the last trial (LT) of Day 1 of habituation training for the preexposed-nonextinguished (PN), preexposed-extinguished (PE), nonpreexposed-nonextinguished (NN), and nonpreexposed-extinguished (NE) groups.

As in the previous experiments, analysis of the 10 startle response amplitudes within each session revealed that there were no within-session response decrements.

Freezing. Figure 4B shows mean percentage of freezing prior to the first trial of each session and on the last trial of Day 1 for all four groups. Overall analysis of first-trial percentage of freezing scores of the PRE and NON-PRE groups across the three sessions of Day 1 revealed significant main effects of session, $F(2,76)=49.39, p<.01$, and group, $F(1$, $38)=12.18, p<.01$, as well as a significant Group $\times$ Session interaction, $F(2,76)=10.31, p<.01$. As in Experiment 2, this interaction reflects the finding that although both groups showed a significant increase in first-trial freezing across sessions on Day 1 ( $p s<.01$ ), the increase exhibited by the NONPRE group was greater than that shown by the PRE group. Groups did not differ in percentage of freezing prior to the initial startle stimulus on Day 1 but did differ significantly by the last trial on Day $1, F(1,38)=12.36, p<.01$. 
As with startle responding, the most sensitive index of the influence of the extinction manipulation on percentage of freezing was the change from last trial of Day 1 to the first trial following extinction training (Trial 1, Day 3). This comparison also is depicted in Figure 4B. Overall analysis of percentage of freezing on the last trial of Day 1 and the first trial of Day 3 for all four groups yielded a significant main effect of group, $F(3,36)=5.06, p<.01$, but the Group $\times$ Session interaction was not significant, $F(3,36)=2.28, p>$ .05. Despite the absence of a significant interaction, these results showed the same overall pattern as the startle response data. Individual group comparisons revealed that whereas the NE group exhibited a significant reduction in percentage of freezing, $t(9)=5.07, p<.01$, the other three groups showed no change in freezing behavior ( $p s>.65$ ).

The influence of extinction training on percentage of freezing is shown in Figure 5. Both PRE and NON-PRE groups exhibited significant reductions in percentage of freezing across sessions of extinction training [NE, $F(2,18)=9.11, p$ $<.01 ; \mathrm{PE}, F(2,18)=4.05, p<.05$ ]. Extinction training was also successful in eliminating group differences in freezing behavior. Although percentage of freezing by the NE group was significantly greater than that for the PE group on the first trial of extinction training, $F(1,18)=6.43, p<.05$, by the last trial the groups no longer differed, $F(1,18)<1.0$.

Percentage of freezing exhibited by both groups prior to presentation of the initial startle stimulus on Day 1 did not differ from that exhibited by the PRE group during preexposure. During preexposure to the startle apparatus, the PRE group showed a mean of $<5 \%$ of freezing during any one mock-trial sampling period and showed no tendency to change freezing behavior within or between preexposure sessions.

As in the previous experiments, changes in first-trial percentage of freezing could be predicted from within-session changes in freezing behavior. The increase in percentage of freezing observed on first-trial scores on Day 1 was again reflected within sessions. The PRE and NON-PRE groups showed an orderly increase in freezing within Session $1(p s<.01)$.

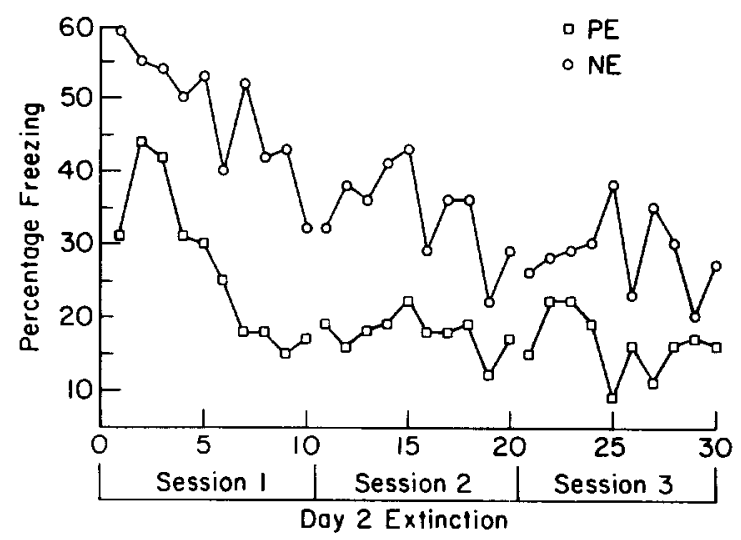

Figure 5. Mean percentage of freezing of the preexposed-extinguished (PE) and nonpreexposed-extinguished (NE) groups on the $\mathbf{3 0}$ trials of extinction training.
The NON-PRE group continued to show an increase in freezing within Session $2(p<.05)$.

\section{Discussion}

The results of Experiment 3 lend further support to the hypothesis that long-term habituation training is accompanied by fear conditioning and that such conditioning inflates startle amplitudes, thereby retarding startle decrements. As in Experiments 1 and 2, comparison of startle responding with percentage of freezing during habituation training indicates that the rate of startle decrement is inversely related to fear conditioning. On Day 1 of habituation training, the nonpreexposed group demonstrated greater development of freezing behavior than the preexposed group, but only the preexposed group exhibited a decrement in startle responding. That the group differences in startle amplitudes following Day 1 of habituation training result from differential fear conditioning is indicated by the effects of the extinction manipulation on both startle amplitudes and percentage of freezing. Giving nonpreexposed animals nonreinforced exposure to the startle apparatus resulted in both the extinction of freezing behavior and the reduction of startle amplitudes to levels exhibited by preexposed animals.

The results of the extinction day also lend support to the proposal that fear conditioning during habituation training stems from the association of startle apparatus cues with the startle stimulus. On the extinction day, exposing animals to the startle apparatus without presentation of the startle stimulus resulted in extinction of the freezing behavior that had developed on Day 1 of habituation training. Because extinction is the consequence of nonreinforced exposure to the conditional stimulus and because Day 1 of habituation training and the extinction day were identical except for presentation of the startle stimulus, one can conclude that apparatus cues and the startle stimulus serve as the conditional and unconditional stimuli, respectively. This perspective is further supported by results of Experiments 2 and 3, which demonstrated that preexposure to the startle chambers did not unconditionally elicit freezing behavior. Therefore, the mere exposure to the startle chambers does not appear to be the unconditional stimulus supporting freezing during habituation training.

\section{General Discussion}

The present study demonstrated that the rate at which the acoustic startle response showed long-term decrements was inversely related to the degree of freezing behavior that developed during long-term habituation training. In all three experiments, manipulations that either retarded development of freezing or reduced its level promoted reductions in startle amplitudes. In Experiment 1, vPAG lesions retarded the development of freezing and accelerated startle response decrements. In Experiment 2, preexposure to the startle chamber produced similar results, reducing the development of freezing while facilitating startle response decrements. In Experiment 3 , an extinction manipulation after the initiation of habituation training reduced both freezing and startle amplitudes. 
We hypothesize that these results are derived from Pavlovian fear conditioning associated with the habituation paradigm. Central to this hypothesis is our contention that development of freezing during habituation training reflects fear conditioning and that fear conditioning elevates startle amplitudes through the response-energizing effects that accompany the central fear state. Fear conditioning is believed to stem from the association of contextual cues of the startle apparatus with the startle stimulus. Contextual cues and the startle stimulus serve as the CS and UCS, respectively. We also assume that presentation of the startle stimulus promotes not only fear conditioning, which enhances startle responding, but also long-term habituation, which functions to reduce startle responding. Consequently, the facilitated long-term startle decrements observed in the experimental groups in the present study are viewed as resulting from the long-term habituation process being relatively unopposed by the response inflating effects associated with the central fear state. For example, conditioned fear was attenuated in Experiment 1 because lesions of the vPAG interfered with the central fear state. In Experiments 2 and 3, however, conditioned fear was reduced because latent inhibition produced by preexposure retarded the development of the CS/UCS association (Experiment 2) and extinction reduced the strength of the CS/UCS interaction (Experiment 3).

In all three experiments, the freezing behavior that developed early in habituation training was extinguished to nearly pretraining levels despite continued pairing of contextual cues with the startle stimulus. The nonmonotonic nature of the freezing function can be explained if it is assumed that with extended presentations the startle stimulus loses its ability not only to elicit a startle response but also to serve as an aversive UCS. Once the startle stimulus loses its properties as a fearinvoking stimulus, habituation trials become equivalent to extinction trials for conditioned fear. This analysis is analogous to the extinction of conditioned emotional response observed when low-shock intensities are employed. Annau and Kamin (1961) reported that rats receiving low-shock intensities during training recover their operant response rates, suggesting adaptation to the aversiveness of the UCS.

Although the startle enhancing and startle decrementing processes that accompany presentation of the startle stimulus interact to determine response strength, these processes are assumed to be independent. In Experiment 3, differences in startle amplitudes that developed between preexposed and nonpreexposed groups were eliminated by extinguishing fear in the nonpreexposed group to the level exhibited by the preexposed group. This outcome indicates that, prior to extinction training, both groups had become similarly habituated to the startle stimulus, despite differences they exhibited in startle amplitudes. The startle inflating effects accompanying the greater fear conditioning in the nonpreexposed group masked the startle decrements associated with the habituation process. Extinguishing fear conditioning reduced the accompanying inflating influence on startle amplitude and revealed the level of habituation to the startle stimulus. Had the differences in startle amplitude exhibited by these groups prior to extinction training been the result of fear conditioning interfering with the habituation process, extinction training would not have affected startle amplitude.

The model of startle response habituation outlined here has basic similarities with the dual-process theory of habituation (Groves \& Thompson, 1970; Thompson \& Spencer, 1966). Both models maintain that repeated elicitation of reflex behavior invokes two independent and opposing processes that interact to determine response amplitude. The habituation process is proposed to underlie response decrements and is opposed by a second process, sensitization, that acts to inflate response amplitudes. According to dual-process theory, the early course of habituation training is accompanied by an increase in both the habituation and sensitization processes, resulting in either no change or a slight increase in response amplitude. As habituation training continues, the sensitization process itself habituates to pretraining levels. As a result, the habituation process is progressively unopposed by the response inflating effects associated with sensitization, and response amplitudes decrease. The conditioned fear observed during long-term habituation training in the present study mimics the form of the sensitization function as described by dual-process theory. As noted here, fear conditioning in all three experiments was initiated with the beginning of habituation training and showed an orderly increase on Day 1. With extended habituation training, fear conditioning was extinguished to pretraining levels. This extinction of fear conditioning would be analogous to the habituation of sensitization outlined by the dual-process theory.

The present model differs from dual-process theory in that we emphasize long-term processes and assume an associative basis for sensitization. Our model of habituation was developed by examining long-term startle decrements, which show no recovery over extended periods of time (e.g., Jordan \& Leaton, 1982; Leaton, 1976). Dual-process theory, on the other hand, was devised through the analysis of short-term response decrements-that is, decrements that recover over intervals of seconds or minutes (e.g., Davis, 1972; Leaton, 1976; Thompson \& Spencer, 1966). Dual-process theory assumes that upon cessation of the eliciting stimulus, both habituation and sensitization spontaneously decay (Groves \& Thompson, 1970). In our conceptualization, sensitization during long-term habituation training is believed to derive from the fear conditioning that accompanies training. Consequently, long-term sensitization does not decay spontaneously but is controlled by associative variables. Just as previous research has shown the existence of independent short-term and long-term habituation processes (Leaton, 1976; Jordan \& Leaton, 1982), the present data in combination with the dual-process theory suggest the existence of independent short-term and long-term sensitization processes.

The influence of associative variables on the rate of longterm response decrements has been described by Wagner $(1976,1978,1979)$. He proposed that long-term habituation reflects the retrieval of a memorial representation of the response-eliciting stimulus from long-term to short-term memory. This "retrieval-generated priming" is purportedly the consequence of exposure to contextual cues (CS) that become associated with the eliciting stimulus (UCS) during 
habituation training. Priming renders the eliciting stimulus less "surprising" and results in a decrement in the amplitude or probability of the response. Within the framework of Wagner's associative theory, preexposure to the contextual cues of the startle apparatus is operationally equivalent to the procedure of latent inhibition. Accordingly, preexposure to the startle apparatus ought to retard formation of the association between contextual cues and the startle stimulus and thereby slow the rate of startle-response decrements. Contrary to Wagner's prediction, Experiments 2 and 3 showed that rather than slowing the rate of startle decrements, preexposure facilitated them. Wagner's theory also predicts that posthabituation exposure to contextual cues in the absence of the startle stimulus will extinguish the association between contextual cues and the startle stimulus, thus extinguishing habituation and restoring startle response amplitudes. Contrary to this prediction, Experiment 3 showed that extinction following initial habituation training reduced startle amplitudes. Although the results of the present study are inconsistent with Wagner's associative theory of habituation, our data do indicate an important role of associative variables in long-term habituation. However, we have shown that it is the sensitization process and not the habituation process that is influenced by the manipulation of associative variables.

Marlin and Miller (1981) and Korn and Moyer (1966) found that preexposure to the startle apparatus lowered the probability and amplitude of the acoustic startle response. Neither group evaluated long-term response decrements following preexposure, but both found no change in short-term decrements. Marlin and Miller (1981) interpreted these findings as indicating that exposure to the novel contextual cues of the startle apparatus aroused the animal and that this arousal elevated startle responding. Preexposure to the startle apparatus allowed animals to habituate to novel apparatus cues, thereby reducing arousal and lowering the probability or amplitude of the startle response. By contrast, Experiments 2 and 3 showed no effect of preexposure on the initial startle amplitude. This discrepancy may be the result of how initial startle responding is evaluated. Marlin and Miller analyzed their data in blocks of 20 trials, making it impossible to determine the effects of preexposure on the response of animals to the first startle stimulus of habituation training. Similarly, Korn and Moyer analyzed their data in blocks of five trials and did not include the initial startle response. These procedures run the risk of confounding initial response levels with changes in the rate of response decrements. Although novel (i.e., dishabituating stimuli; Groves \& Thompson, 1970) or intense (e.g., loud background noise; Davis, 1974a, 1974b) contextual cues have been shown to sensitize the acoustic startle response, there is no evidence that the novelty of the startle apparatus used in the present study was sufficient to inflate startle amplitudes. Furthermore, to the extent that freezing behavior during preexposure provides an index of the arousing/sensitizing effects of exposure to novel apparatus cues, we found no evidence for such an influence.

The present study has outlined the relation between longterm acoustic startle decrements and the conditioned fear that develops during habituation training. We propose that this relation can be best incorporated within a dual-process theory of long-term habituation. Fear conditioning is viewed as reflecting an associatively based long-term sensitization process, which interacts with a long-term habituation process to determine response strength. This conceptualization has implications for how differences in startle decrements are interpreted. In the present study, the accelerated startle decrements exhibited by animals with vPAG lesions and animals preexposed to the startle apparatus were not viewed as resulting from enhanced habituation, but rather from retarded development of sensitization. Any manipulation, whether behavioral or neurological, that appears to alter long-term habituation must be examined for its possible influence on longterm sensitization.

\section{References}

Annau, Z., \& Kamin, L. J. (1961). The conditioned emotional response as a function of intensity of the U.S. Journal of Comparative and Physiological Psychology, 54, 428-432.

Beitz, A. J. (1982). The organization of afferent projections to the midbrain periaqueductal gray of the rat. Neuroscience, 51, 133159.

Blanchard, R. J., \& Blanchard, D. C. (1969). Crouching as an index of fear. Journal of Comparative and Physiological Psychology, 67, 307-375.

Bolles, R. C., \& Collier, A. C. (1976). Effect of predictive cues on freezing in rats. Animal Learning \& Behavior, 4, 6-8.

Brown, J. S. (1939). A note on a temporal gradient of reinforcement. Journal of Experimental Psychology, 25, 211-227.

Brown, J. S., Kalish, H. I., \& Farber, I. E. (1951). Conditioned fear as revealed by magnitude of startle response to an auditory stimulus. Journal of Experimental Psychology, 41, 317-328.

Davis, M. (1972), Differential retention of sensitization and habituation of the startle response in the rat. Journal of Comparative and Physiological Psychology, 78, 260-267.

Davis, M. (1974a). Sensitization of the rat acoustic startle response by noise. Journal of Comparative and Physiological Psychology, 87, 571-581.

Davis, M. (1974b). Signal-to-noise ratio as a predictor of startle amplitude and habituation in the rat. Journal of Comparative and Physiological Psychology, 86, 812-825.

Davis, M., \& Astrachan, D. I. (1978). Conditioned fear and startle magnitude: Effects of different footshock or backshock intensities used in training. Journal of Experimental Psychology: Animal Behavior Processes, 4, 95-103.

Fanselow, M. S. (1980). Conditional and unconditional components of post-shock freezing. Pavlovian Journal of Biological Sciences, 15, 177-182.

Fanselow, M. S. (1981). Naloxone and Pavlovian fear conditioning. Learning and Motivation, 12, 398-419.

Frommer, G. P. (1971). Modified nontraumatic headholder. Behavioral Research Methods \& Instrumentation, 3, 225.

Groves, P. M., \& Thompson, R. F. (1970). Habituation: A dualprocess theory. Psychological Review, 77, 419-450.

Halpern, M. (1968). Effects of midbrain central gray lesions on escape-avoidance behavior in rats. Physiology \& Behavior, 3, 171178.

Jordan, W. P., \& Leaton, R. N. (1982). Effects of mesencephalic reticular formation lesions on habituation of startle and lick suppression responses in the rat. Journal of Comparative and Physiological Psychology, 96, 170-183. 
Korn, J. H., \& Moyer, K. E. (1966). Habituation of the startle response and of heart rate in the rat. Canadian Journal of Psychology, 20, 183-190.

Kurtz, K. H., \& Siegel, A. (1966). Conditioned fear and magnitude of startle response: A replication and extension. Journal of Comparative and Physiological Psychology, 62, 8-14.

Leaton, R. N. (1976). Long-term retention of the habituation of lick suppression and startle response produced by a single auditory stimulus. Journal of Experimental Psychology: Animal Behavior Processes, 2, 248-259.

Leaton, R. N., \& Borszcz, G. S. (1985). Potentiated startle: Its relation to freezing and shock intensity in rats. Journal of Experimental Psychology: Animal Behavior Processes, 11, 421-428.

Liebman, J. M., Mayer, D. J., \& Liebeskind, J. C. (1970). Mesencephalic central gray lesions and fear-motivated behavior in rats. Brain Research, 23, 353-370.

Lubow, R. E., \& Moore, A. U. (1959). Latent inhibition: The effect of nonreinforced preexposure to the conditioned stimulus. Journal of Comparative and Physiological Psychology, 52, 415-419.

Lubow, R. E., Rifkin, B., \& Alek, M. (1976). The context effect: The relationship between stimulus preexposure and environmental preexposure determines subsequent learning. Journal of Experimental Psychology: Animal Behavior Processes, 2, 38-47.

Lyon, M. (1964). The role of central midbrain structures in conditioned responding to aversive noise in the rat. Journal of Compar- ative Neurology, 122, 407-429.

Marlin, N. A., \& Miller, R. R. (1981). Associations to contextual stimuli as a determinant of long-term habituation. Journal of Experimental Psychology: Animal Behavior Processes, 7, 313-333.

Pellegrino, L. J., \& Cushman, A. J. (1967). A stereotaxic atlas of the rat brain. New York: Appelton-Century-Crofts.

Thompson, R. F., \& Spencer, W. A. (1966). Habituation: A model phenomenon for the study of neuronal substrates of behavior. Psychological Reviews, 73, 16-43.

Wagner, A. R. (1976). Priming in STM: An information-processing mechanism for self-generated or retrieval-generated depression in performance. In T. J. Tighe \& R. N. Leaton (Eds.), Habituation: Perspectives from child development, animal behavior, and neurophysiology, (pp. 95-128). Hillsdale, NJ: Erlbaum.

Wagner, A. R. (1978). Expectancies and the priming of STM. In S. H. Hulse, H. Fowler, \& W. K. Honig (Eds.), Cognitive processes in animal behavior (pp. 177-209). Hillsdale, NJ: Erlbaum.

Wagner, A. R. (1979). Habituation and memory. In A. Dickinson \& R. A. Boakes (Eds.), Mechanisms of learning and motivation (pp. 53-82). Hillsdale, NJ: Erlbaum.

Received March 2, 1988

Revision received May 30, 1988

Accepted June 6, 1988

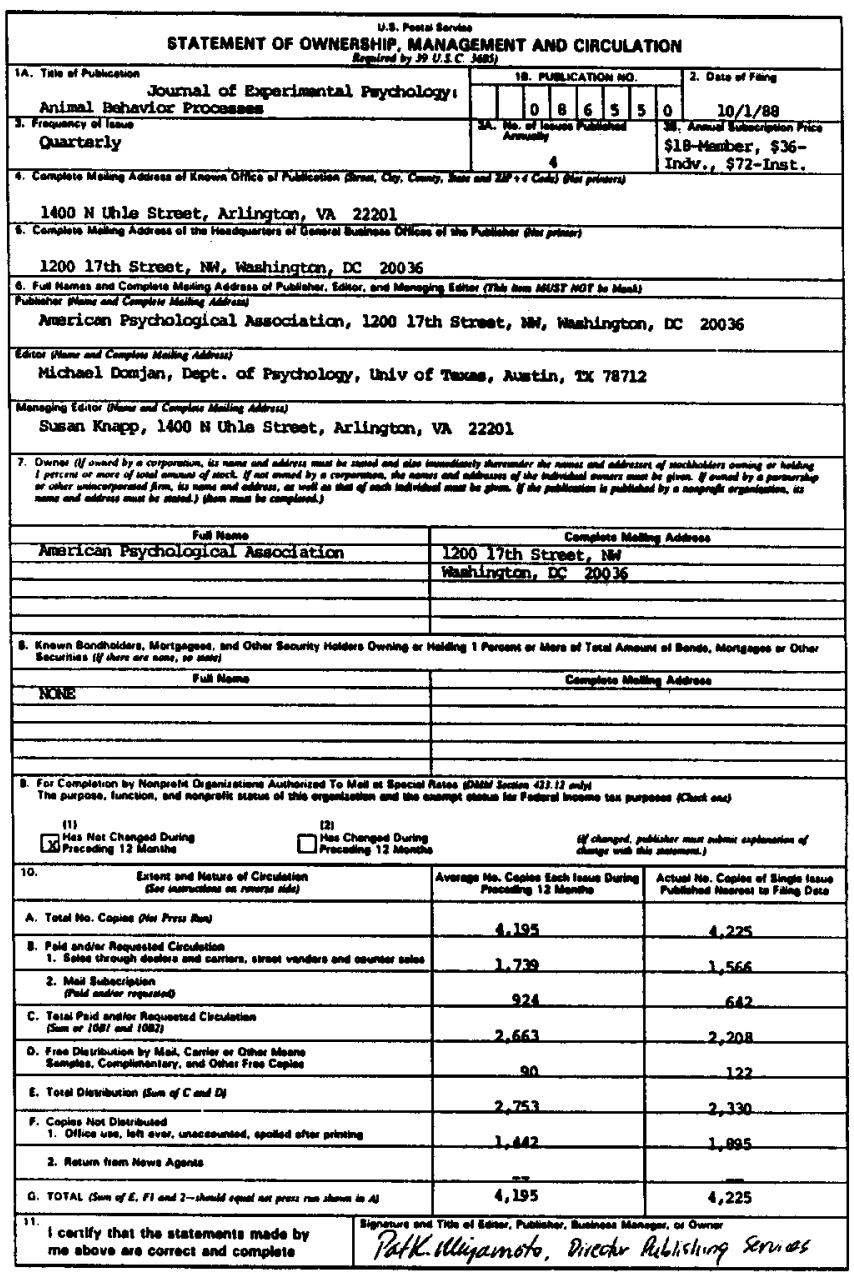

